\title{
Pseudo-Hermiticity, weak pseudo-Hermiticity and $\eta$-orthogonality condition
}

\author{
B. Bagchi ${ }^{a, *}$, C. Quesne ${ }^{b, \dagger}$ \\ a Department of Applied Mathematics, University of Calcutta, \\ 92 Acharya Prafulla Chandra Road, Calcutta 700 009, India \\ ${ }^{b}$ Physique Nucléaire Théorique et Physique Mathématique, Université Libre de Bruxelles, \\ Campus de la Plaine CP229, Boulevard du Triomphe, B-1050 Brussels, Belgium
}

\begin{abstract}
We discuss certain features of pseudo-Hermiticity and weak pseudo-Hermiticity conditions and point out that, contrary to a recent claim, there is no inconsistency if the correct orthogonality condition is used for the class of pseudo-Hermitian, PTsymmetric Hamiltonians of the type $H_{\beta}=[p+\mathrm{i} \beta \nu(x)]^{2} / 2 m+V(x)$.
\end{abstract}

PACS: 03.65.Ca

Keywords: Non-Hermitian Hamiltonians; PT Symmetry; Pseudo-Hermiticity; Supersymmetric Quantum Mechanics

Corresponding author: C. Quesne, Physique Nucléaire Théorique et Physique Mathématique, Université Libre de Bruxelles, Campus de la Plaine CP229, Boulevard du Triomphe, B-1050 Brussels, Belgium

Telephone: 32-2-6505559

Fax: 32-2-6505045

E-mail: cquesne@ulb.ac.be

*E-mail: bbagchi@cucc.ernet.in

${ }^{\dagger}$ Directeur de recherches FNRS; E-mail: cquesne@ulb.ac.be 
In recent times it has been stressed that neither Hermiticity nor PT symmetry serves as a necessary condition for a quantum Hamiltonian to preserve the reality of its bound-state

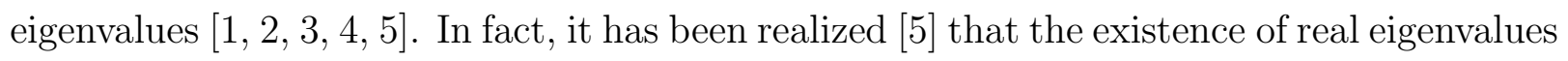
can be associated with a non-Hermitian Hamiltonian provided it is $\eta$-pseudo-Hermitian:

$$
\eta H=H^{\dagger} \eta
$$

where $\eta$ is a Hermitian linear automorphism and, assuming $\hbar=2 m=1$,

$$
H=p^{2}+V(x)
$$

for $V(x) \in \mathbb{C}$ and $p=-\mathrm{i} \partial_{x}$. Then, in such a case, the spectrum of a diagonalizable $H$ is real if there exists a linear invertible operator $O$ such that $\eta=\left(O O^{\dagger}\right)^{-1}$. Moreover, one can relax $H$ to be only weak pseudo-Hermitian [6] by not restricting $\eta$ to be Hermitian.

The purpose of this Letter is to establish the following results:

(i) The twin concepts of pseudo-Hermiticity and weak pseudo-Hermiticity are complementary to one another.

(ii) For a first-order differential realization, $\eta$ may be anti-Hermitian but for the secondorder case, $\eta$ is necessarily Hermitian. For both cases, we make connections to the same PT-symmetric Scarf II Hamiltonian (having normalizable eigenfunctions) to show that the choice of $\eta$ is not unique in ascertaining the character of the Hamiltonian.

(iii) For the class of $\eta$-pseudo-Hermitian, PT-symmetric Hamiltonians described by [0, 8] 凹

$$
H_{\beta}=[p+\mathrm{i} \beta \nu(x)]^{2}+V(x), \quad \beta \in \mathbb{R},
$$

where the odd function $\nu(x) \in \mathbb{R}, V(x)$ is PT-symmetric, and

$$
\eta=\exp \left[-2 \beta \int^{x} \nu(y) d y\right]
$$

our earlier derivation [9] of the generalized continuity equation for Hamiltonians of the form (2) [with $V(x)$ PT-symmetric] can be extended to $H_{\beta}$ as well. The resulting $\eta$ orthogonality condition needs to be implemented judiciously.

\footnotetext{
${ }^{1}$ Note that in Ref. [8], it is assumed that $\hbar=m=1$ instead of $\hbar=2 m=1$.
} 
We begin by addressing to the point (i) above. Consider some non-Hermitian $\eta$ that is subject to the condition (11). Taking Hermitian conjugate, we obtain, on adding and subtracting, the following combinations

$$
\eta_{+} H=H^{\dagger} \eta_{+}, \quad \eta_{-} H=H^{\dagger} \eta_{-}
$$

where $\eta_{ \pm}=\eta \pm \eta^{\dagger}$. While the first one of (5) corresponds to strict pseudo-Hermiticity, the second one points to weak pseudo-Hermiticity with a new anti-Hermitian operator $\eta_{-}$. Note that $\eta_{+}$is Hermitian. It is thus clear that weak pseudo-Hermiticity is not more general than pseudo-Hermiticity but works complementary to it.

We now turn to (ii). Decomposing $V(x)$ and $\eta$ as

$$
\begin{aligned}
V(x) & =V_{R}(x)+\mathrm{i} V_{I}(x), \\
\eta & =\frac{d}{d x}+f(x)+\mathrm{i} g(x),
\end{aligned}
$$

where $V_{R}, V_{I}, f, g \in \mathbb{R}$, we get, on inserting (6) into (1), the relations

$$
\begin{aligned}
V_{I} & =\mathrm{i}\left(f^{\prime}+\mathrm{i} g^{\prime}\right), \\
V_{R}^{\prime}+\mathrm{i} V_{I}^{\prime} & =-\left(f^{\prime \prime}+\mathrm{i} g^{\prime \prime}\right)-2 \mathrm{i} V_{I}(f+\mathrm{i} g) .
\end{aligned}
$$

In (7) the primes denote the order of differentiations with respect to the variable $x$. We are then led to the conditions

$$
V_{R}^{\prime}=-2 g g^{\prime}, \quad c g^{\prime}=0, \quad c \in \mathbb{R},
$$

which imply the existence of two solutions corresponding to $c=0$ and $g^{\prime}=0$, respectively. In the following we concentrate on the case $c=0$ because $g^{\prime}=0$ yields a trivial result that corresponds to a real constant potential with no normalizable eigenfunction.

For the choice $c=0$, it turns out that

$$
f=0, \quad V_{R}=-g^{2}+k, \quad V_{I}=-g^{\prime},
$$

where $k \in \mathbb{R}$. In consequence, we have the results

$$
\begin{aligned}
V(x) & =-g^{2}(x)+k-\mathrm{i} g^{\prime}(x), \\
\eta & =\frac{d}{d x}+\mathrm{i} g(x) .
\end{aligned}
$$


The above form of $V(x)$ shows that, in the framework of supersymmetric quantum mechanics, we can associate to it an imaginary superpotential $W(x)=\mathrm{i} g(x)$, its partner being the complex conjugate potential. We also observe that, for even $g$ functions, the representation of $\eta$ makes it anti-Hermitian in character. Let us consider the following specific example for $g=d \operatorname{sech} x, d \in \mathbb{R}$. We get from (10)

$$
\begin{aligned}
V(x) & =-d^{2} \operatorname{sech}^{2} x+k+\mathrm{i} d \operatorname{sech} x \tanh x, \\
\eta & =\frac{d}{d x}+\mathrm{i} d \operatorname{sech} x
\end{aligned}
$$

It is obvious that $V(x)$ is a particular case of the generalized PT-symmetric Scarf II potential investigated previously by us [10] in connection with the complex algebra $\operatorname{sl}(2, \mathbb{C})$. A comparison with the results obtained there shows that, in the present case, we have a single series of real eigenvalues with normalizable eigenfunctions provided $d>\frac{1}{2}$. The corresponding Hamiltonian is both P-pseudo-Hermitian and $\eta$-weak-pseudo-Hermitian with $\eta$ given by (11). Our example confirms the assertion [11] that, for a given non-Hermitian Hamiltonian, there could be infinitely many $\eta$ satisfying the weak-pseudo-Hermiticity or the pseudo-Hermiticity condition.

We next attend to a second-order differential representation of $\eta$ :

$$
\eta=\frac{d^{2}}{d x^{2}}-2 p(x) \frac{d}{d x}+b(x),
$$

where $p, b \in \mathbb{C}$. Substituting (12) into the condition (11), we obtain the constraints

$$
\begin{aligned}
b & =-p^{\prime}+p^{2}-\frac{p^{\prime \prime}}{2 p}+\left(\frac{p^{\prime}}{2 p}\right)^{2}+\frac{\gamma}{4 p^{2}}, \\
V & =2 p^{\prime}+p^{2}+\frac{p^{\prime \prime}}{2 p}-\left(\frac{p^{\prime}}{2 p}\right)^{2}-\frac{\gamma}{4 p^{2}}-\delta, \\
V^{*} & =-2 p^{\prime}+p^{2}+\frac{p^{\prime \prime}}{2 p}-\left(\frac{p^{\prime}}{2 p}\right)^{2}-\frac{\gamma}{4 p^{2}}-\delta,
\end{aligned}
$$

where $\gamma, \delta \in \mathbb{R}$. From the last two relations in (13), it is clear that $p(x)$ must be pure imaginary,

$$
p(x)=\mathrm{i} a(x),
$$


where $a(x) \in \mathbb{R}$. As such $V(x)$ and $\eta$ acquire the forms

$$
\begin{aligned}
V(x) & =2 \mathrm{i} a^{\prime}-a^{2}+\frac{a^{\prime \prime}}{2 a}-\left(\frac{a^{\prime}}{2 a}\right)^{2}+\frac{\gamma}{4 a^{2}}-\delta \\
\eta & =\frac{d^{2}}{d x^{2}}-2 \mathrm{i} a(x) \frac{d}{d x}+b(x),
\end{aligned}
$$

with $b(x)=-V(x)+\mathrm{i} a^{\prime}-2 a^{2}-\delta$. In (15), $\eta$ can be easily recognized to be a Hermitian operator since it can be written in the form $\eta=-\tilde{O}^{\dagger} \tilde{O}$, where $\tilde{O}=\frac{d}{d x}+r-\mathrm{i} a$, $\tilde{O}^{\dagger}=$ $-\frac{d}{d x}+r+\mathrm{i} a$, and $r^{2}-r^{\prime}=\frac{a^{\prime \prime}}{2 a}-\left(\frac{a^{\prime}}{2 a}\right)^{2}+\frac{\gamma}{4 a^{2}}$. In Ref. [12], such a decomposition of $\eta$ was assumed, a priori, to arrive at some non-Hermitian Hamiltonians with real spectra.

Let us, however, confine ourselves to the following choice

$$
a(x)=-\frac{1}{2} B(2 A+1) \operatorname{sech} x, \quad \gamma=0, \quad \delta=\frac{1}{4},
$$

where $A+\frac{1}{2}>0, B>0$, and $A-B+\frac{1}{2}$ is not an integer. We are again led to the PTsymmetric Scarf II potential having a more general form than obtained with the first-order differential realization of $\eta$ :

$$
V(x)=-V_{1} \operatorname{sech}^{2} x-\mathrm{i} V_{2} \operatorname{sech} x \tanh x,
$$

where $V_{1}=\frac{1}{4}\left[B^{2}(2 A+1)^{2}+3\right]>0$ and $V_{2}=-B(2 A+1) \neq 0$. According to Refs. [13, 14, the condition for real eigenvalues for the Hamiltonian corresponding to (17) is $\left|V_{2}\right| \leq V_{1}+\frac{1}{4}$. Here it amounts to $[B(2 A+1)-2]^{2} \geq 0$, which is always met.

Of particular interest is the special case $B=1$ :

$$
V(x)=-\left(A^{2}+A+1\right) \operatorname{sech}^{2} x+\mathrm{i}(2 A+1) \operatorname{sech} x \tanh x .
$$

On setting $A+\frac{1}{2}=-\lambda(\lambda<0)$, Eq. (18) can be seen to reduce to the potential $V^{(1)}-\frac{1}{4}$ of Ref. [15] for $\mu=1$. The associated energy levels of (18) are [10]: $E_{n}^{(-\lambda)}=-\left(\lambda+n+\frac{1}{2}\right)^{2}$ and coincide with $E_{n}^{(2)}-\frac{1}{4}$ of [15]. Note that there is, in general, a doubling of energy levels in transiting from the real to the PT-symmetric Scarf II potential. In fact, the second algebra of $\operatorname{sl}(2, \mathbb{C})$ leads to an additional energy level $E_{0}^{(1)}=-\frac{1}{4}$ that is consistent with the zero-energy state of [15]. 
Finally, we take up a general derivation of the continuity equation for the class of Hamiltonians $H_{\beta}$ given by (3). The associated Schrödinger equation reads

$$
\mathrm{i} \frac{\partial \psi(x, t)}{\partial t}=-\left(-\frac{\partial}{\partial x}+\beta \nu(x)\right)^{2} \psi(x, t)+V(x) \psi(x, t) .
$$

From this it follows that the function $\psi^{*}(-x, t)$ satisfies

$$
-\mathrm{i} \frac{\partial \psi^{*}(-x, t)}{\partial t}=-\left(-\frac{\partial}{\partial x}+\beta \nu(x)\right)^{2} \psi^{*}(-x, t)+V(x) \psi^{*}(-x, t) .
$$

On considering Eq. (19) for some solution $\psi_{1}(x, t)$ and Eq. (20) for some other solution $\psi_{2}(x, t)$ and then multiplying (19) and (20) by $\exp \left[-2 \beta \int^{x} \nu(y) d y\right] \psi_{2}^{*}(-x, t)$ and $\exp \left[-2 \beta \int^{x} \nu(y) d y\right] \psi_{1}(x, t)$, respectively, we obtain, on subtracting, a natural generalization of the continuity equation for PT-symmetric quantum mechanics to its $\eta$-pseudo-Hermitian extension, namely

$$
\frac{\partial P_{\eta}(x, t)}{\partial t}+\frac{\partial J_{\eta}(x, t)}{\partial x}=0
$$

where

$$
\begin{aligned}
P_{\eta}(x, t) & =\eta \psi_{2}^{*}(-x, t) \psi_{1}(x, t) \\
J_{\eta}(x, t) & =\frac{\eta}{\mathrm{i}}\left[\psi_{2}^{*}(-x, t) \frac{\partial \psi_{1}(x, t)}{d x}-\psi_{1}(x, t) \frac{\partial \psi_{2}^{*}(-x, t)}{d x}\right]
\end{aligned}
$$

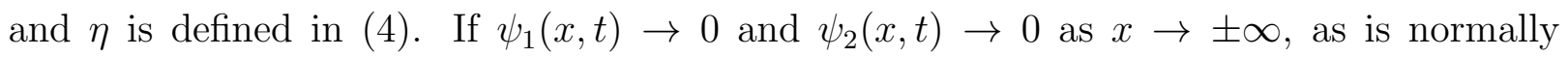
expected for bound-state wave functions, then integration of (21) over the entire real line gives the conservation law

$$
\frac{\partial}{\partial t} \int_{-\infty}^{\infty} d x \eta \psi_{2}^{*}(-x, t) \psi_{1}(x, t)=0
$$

In the case of energy eigenfunctions

$$
\psi_{1}(x, t)=u_{1}(x) e^{-\mathrm{i} E_{1} t}, \quad \psi_{2}(x, t)=u_{2}(x) e^{-\mathrm{i} E_{2} t},
$$

corresponding to the eigenvalues $E_{1}$ and $E_{2}$ respectively, Eq. (23) reduces to

$$
\left(E_{1}-E_{2}^{*}\right) \int_{-\infty}^{\infty} d x \eta u_{2}^{*}(-x) u_{1}(x)=0
$$


Equation (25) represents the $\eta$-orthogonality condition [5]. Obviously it transforms to the PT-orthogonality [9, [16]

$$
\left(E_{1}-E_{2}^{*}\right) \int_{-\infty}^{\infty} d x u_{2}^{*}(-x) u_{1}(x)=0
$$

for $\nu(x)=0$. Indeed Eq. (25) can be derived from (26) by effecting a gauge transformation on the wave functions $u$ of $H$ in a manner $u \rightarrow \exp \left[-\int^{x} \beta \nu(y) d y\right] u, V(x)$ being PTsymmetric. As such $H_{\beta}$ may be looked upon as a gauge-transformed version of $H$. However, it needs to be emphasized that care should be taken to correctly implement the normalization conditions deriving from (25) and (26) and which are appropriate to the Hamiltonians $H_{\beta}$ and $H$, respectively. Thus although PT-symmetric, the form of the $\eta$-pseudo-Hermitian Hamiltonian $H_{\beta}$ at once suggests that the normalization condition related to (25) is to be used rather than that connected with (26), a point overlooked in Ref. [8].

In summary, we have shown that $\eta$-pseudo-Hermiticity and weak pseudo-Hermiticity are essentially complementary concepts. We have provided an explicit example of PTsymmetric Scarf II model to demonstrate that $\eta$ does not necessarily have a unique representation to determine the character of the associated non-Hermitian Hamiltonian. We have also pointed out the correct use of the $\eta$-orthogonality condition when dealing with a pseudo-Hermitian gauge-transformed Hamiltonian. 


\section{References}

[1] D. Bessis, unpublished (1992).

[2] C.M. Bender, S. Boettcher, Phys. Rev. Lett. 80 (1998) 5243.

[3] C.M. Bender, S. Boettcher, P.N. Meisinger, J. Math. Phys. 40 (1999) 2201.

[4] P. Dorey, C. Dunning, R. Tateo, J. Phys. A 34 (2001) 5679.

[5] A. Mostafazadeh, J. Math. Phys. 43 (2002) 205; 43 (2002) 2814.

[6] L. Solombrino, Weak pseudo-Hermiticity and antilinear commutant, Preprint quant$\mathrm{ph} / 0203101$.

[7] A. Mostafazadeh, On the pseudo-Hermiticity of general PT-symmetric standard Hamiltonians in one dimension, Preprint math-ph/0204013.

[8] Z. Ahmed, Phys. Lett. A 294 (2002) 287.

[9] B. Bagchi, C. Quesne, M. Znojil, Mod. Phys. Lett. A 16 (2001) 2047.

[10] B. Bagchi, C. Quesne, Phys. Lett. A 273 (2000) 285.

[11] A. Mostafazadeh, Pseudo-supersymmetric quantum mechanics and isospectral pseudoHermitian Hamiltonians, Preprint math-ph/0203041.

[12] T.V. Fityo, A new class of non-Hermitian Hamiltonians with real spectra, Preprint quant-ph/0204029.

[13] Z. Ahmed, Phys. Lett. A 282 (2001) 343; 287 (2001) 295.

[14] B. Bagchi, C. Quesne, Non-Hermitian Hamiltonians with real and complex eigenvalues in a Lie-algebraic framework, Preprint math-ph/0205002, to be published in Phys. Lett. A.

[15] B. Bagchi, R. Roychoudhury, J. Phys. A 33 (2000) L1.

[16] G.S. Japaridze, J. Phys. A 35 (2002) 1709. 